\title{
24-h ambulatory blood pressure and retinopathy in normoalbuminuric IDDM patients
}

\author{
P. L. Poulsen ${ }^{1}$, T. Bek ${ }^{2}$, E. Ebbehloj ${ }^{1}$, K.W.Hansen ${ }^{1}$, C. E. Mogensen ${ }^{1}$ \\ ${ }^{1}$ Medical Department M (Diabetes and Endocrinology) Aarhus University Hospital, Denmark \\ ${ }^{2}$ Eye Department, Aarhus University Hospital, Denmark
}

Summary The role of blood pressure elevation in the incidence and progression of diabetic retinopathy is not clearly established and results have been conflicting. Blood pressure and urinary albumin excretion (UAE) are closely related. In order to evaluate the independent relationship between retinopathy and blood pressure elevation, precise information on UAE is essential, as confounding by renal disease (incipient or overt), cannot otherwise be excluded.The aim of the present study was to evaluate the association between diabetic retinopathy and 24-h ambulatory blood pressure (AMBP) in a group of well-characterized normoalbuminuric IDDM patients. In 65 normoalbuminuric (UAE $<20 \mu \mathrm{g} / \mathrm{min}$ ) IDDM patients we performed 24-h AMBP (Spacelabs 90 207) with readings at 20-min intervals. Fundus photographs were graded independently by two experienced ophthalmologists. UAE was measured by RIA and expressed as geometric mean of three overnight collections made within 1 week. $\mathrm{HbA}_{1 \mathrm{c}}$ was determined by HPLC. Tobacco use and level of physical activity were assessed by questionnaire. Fifteen patients had no detectable retinal changes [grade 1], 35 had grade 2 retinopathy; and 15 had more advanced retinopathy [grade 3--6]. Diastolic night blood pressure was significantly higher in patients with diabetic retinopathy compared to patients without retinopathy $(68 \pm 8 \mathrm{mmHg}$ [grade 3--6] and $65 \pm 6 \mathrm{mmHg}$ [grade 2], compared to $61 \pm 4 \mathrm{mmHg}$ [grade 1], $p=0.02$ ). Diurnal blood pressure variation was significantly blunted in the patients with retinopathy as indicated by a higher night/day ratio of diastolic blood pressure $(84.6 \% \pm 4$ [grade 3--6], and $81.2 \% \pm 6$ [grade 2] compared to $79.1 \% \pm 4$ [grade $1], p=0.01)$. Heart rate tended to be higher in patients in group 2 and 3--6 compared to patients without retinopathy with $p$ values of 0.07 and 0.11 for day-time and $24 \mathrm{~h}$ values, respectively. Mean $\mathrm{HbA}_{1 \mathrm{c}}$ increased significantly with increasing levels of retinopathy $(p<0.01)$. Patients were similar regarding sex, age, tobacco use, and level of physical activity. Notably, UAE was almost identical in the three groups $(5.0 \times / \div 1.7$ [grade 1], $3.9 \times / \div 1.8$ [grade 2], and $5.1 \times / \div 1.6 \mu \mathrm{g} / \mathrm{min}$ [grade $3--6]$ ). In conclusion, night blood pressure is higher and circadian blood pressure variation blunted in patients with retinopathy compared to patients without retinopathy despite strict normoalbuminuria and similar UAE levels in the groups compared. Our data suggest that the association between blood pressure and diabetic retinopathy is present also when coexisting renal disease is excluded. Disturbed diurnal variation of blood pressure is a pathophysiological feature related to the development of both retinopathy and nephropathy in IDDM patients. [Diabetologia (1998) 41: 105--110]

Keywords Diabetic retinopathy, 24-h ambulatory blood pressure, IDDM, urinary albumin excretion.
Received: 27 May 1997 and in revised form: 5 September 1997

Corresponding author: Dr. P. L. Poulsen, Department of Medicine M (Diabetes and Endocrinology), Aarhus Kommunehospital, DK-8000 Aarhus C, Denmark
Abbreviations: IDDM, Insulin-dependent diabetes mellitus; UAE, urinary albumin excretion; AMBP, ambulatory blood pressure; N/D ratio, night/day ratio. 
Diabetic retinopathy can be expected to develop in the majority of insulin-dependent diabetic (IDDM) patients [1--4], and despite the beneficial effect of photocoagulation, this complication remains a leading cause of visual loss in young adults [5,6]. Duration of diabetes and poor blood glucose control are established risk factors for the development of retinopathy [7--10], but the pathogenetic mechanisms underlying the initiation and progression of this complication are still poorly understood. Identification of other potentially modifiable clinical parameters associated with retinopathy, e.g. high blood pressure might have implications for understanding pathophysiology, and for preventing and managing retinopathy.

In diabetic nephropathy the close association between blood pressure increase and progression of kidney disease is now clearly established with important consequences for clinical practice: Antihypertensive treatment especially with angiotensin converting enzyme (ACE)-inhibitors has been demonstrated to impede progression of diabetic nephropathy [11-17]. In contrast, the role of blood pressure elevation for the incidence and progression of diabetic retinopathy has not been clearly demonstrated [18--20]. Several studies have been hampered by inadequate methods of establishing the presence and severity of retinopathy as well as insensitive assessment of systemic blood pressure. Furthermore, the characterization of patients with regard to urinary albumin excretion (UAE) has often been insufficient. The association between blood pressure and even minor elevations of UAE is well described [21--29]. Evaluation of a possible independent relationship between retinopathy and blood pressure elevation is rendered impossible without precise information on albumin excretion, since confounding by coexisting incipient renal disease cannot be ruled out.

The aim of the present study was to evaluate the association between diabetic retinopathy and $24 \mathrm{~h}$ ambulatory blood pressure as well as circadian blood pressure variation in a group of well-characterized normoalbuminuric IDDM patients.

\section{Subjects and methods}

Patients. We selected 65 patients from among 120 patients participating in an ongoing prospective study addressing identification of risk factors for the development of complications in IDDM. The following criteria were applied: participants had to be normoalbuminuric (UAE $<20 \mu \mathrm{g} / \mathrm{min}$ in at least two out of three overnight urine collections) and without other chronic diseases. Retinal examination had to be performed within 6 months of the assessment of UAE and blood pressure. None received (or had earlier received) antihypertensive or other medical treatment apart from insulin. UAE was measured by RIA and expressed as geometric mean of three overnight collections made within 1 week. $\mathrm{HbA}_{1 \mathrm{c}}$ was determined by HPLC (non-diabetic range 4.4--6.4\%). Blood glucose was determined by Reflolux II, Boehringer Mannheim (Mannheim, Germany).

Patients were classified according to participation in leisure-time physical activity as: Passive (not participants), Moderate (physical exercise once or twice a week), and Active (Physical exercise more than twice a week). Tobacco consumption was graded as Non-smokers (without daily use of tobacco for at least the last year), Moderate smokers (less than 15 cigarettes per day) and Heavy smokers (More than 15 cigarettes per day).

The study was approved by the local ethics committee and patients gave their written informed consent.

Grading of retinopathy. After induction of cycloplegia and mydriasis by phenylephrin $10 \%$ and tropicamid $1 \%$ eye drops, fundus photography was performed with a Canon 60UV fundus camera on Kodak ectachrome 64 colour diapositive film. In each eye a standard photograph of 60 degrees was taken centered on the foveal region, thus covering and area corresponding approximately to fields $1--5$ of the standard fields used in the Early Treatment For Diabetic Retinopathy Study [30]. For the grading each photograph was projected to a size of $1 \times 1$ meter on a wall board. The number of each type of pathological lesion, haemorrhages and/or microaneurysms, hard exudates, or cotton wool spots was counted (truncated at 99), and the presence of laser scars or vascular abnormalities such as intraretinal microvascular abnormalities (IRMA vessels), venous beading, or neovascularizations was noted. Furthermore, the presence of diabetic maculopathy was noted, defined as hard exudates within one disk diameter of the fovea. Each photograph was evaluated independently by two experienced graders. When the two evaluations of a photograph were discrepant it was reassessed by the two graders together. In case there was still discrepancy the opinion of the most senior grader was used.

On the basis of the grading of all lesions on a photograph each eye was assigned an overall retinopathy grade on a scale from 1--6 according to the principles used in the Wisconsin Epidemiologic Study of Diabetic Retinopathy [1] with a modification to ensure that lesions implying the same risk of progression to proliferative diabetic retinopathy resulted in the same retinopathy level (ETDRS Report 12 [31]). Consequently, the assignment of retinopathy grade was made according to the following guidelines:

1) No retinopathy. 2) a. Less than 20 haemorrhages and/or microaneurysms, or b. Cotton wool spots alone. 3) a. More than or $=20$ haemorrhages and/or microaneurysms, or $b$. Hard exudates combined with any number of haemorrhages and/or microaneurysms, or c. Less than 5 cotton wool spots combined with haemorrhages and/or microaneurysms or hard exudates. 4) More than or $=5$ cotton wool spots or IRMA vessels combined with haemorrhages and/or microaneurysms with or without hard exudates. 5) Venous beading combined with haemorrhages and/or microaneurysms with or without hard exudates, IRMA vessels or cotton wool spots. 6) Proliferative diabetic retinopathy, or scars of photocoagulation known to have been directed at new vessels.

In each patient the retinopathy grade on the worst eye was used for the analysis. The ophthalmologists had no knowledge of the subjects' blood pressure or their glycaemic control and, thus, graded the eye findings in an unbiased way.

24- $h$ blood pressure measurements and circadian blood pressure variation. Ambulatory blood pressure (AMBP) was measured by an oscillometric technique (Spacelabs 90 207, validated by the British Hypertension Society [32]). Readings were 
Table 1. Clinical characteristics of patients grouped according to severity of retinopathy

\begin{tabular}{|c|c|c|c|c|c|c|c|c|c|c|c|}
\hline \multicolumn{2}{|c|}{$\begin{array}{l}\text { Eye } \\
\text { category } n\end{array}$} & $\begin{array}{l}\text { Sex } \\
\left(\% \sigma^{\prime}\right)\end{array}$ & $\begin{array}{l}\text { Age } \\
\text { (years) }\end{array}$ & $\begin{array}{l}\text { Duration } \\
\text { (years) }\end{array}$ & $\begin{array}{l}\mathrm{HbA}_{1 \mathrm{c}} \\
(\%)\end{array}$ & $\begin{array}{l}\text { Insulin } \\
\text { (U/kg body } \\
\text { weight) }\end{array}$ & $\begin{array}{l}\text { UAE } \\
(\mu \mathrm{g} / \mathrm{min} \times / \\
\div \mathrm{TF})\end{array}$ & $\begin{array}{l}\text { Height } \\
(\mathrm{m})\end{array}$ & $\begin{array}{l}\text { Weight } \\
(\mathrm{kg})\end{array}$ & $\begin{array}{l}\text { Smoking } \\
\text { (Non-smoking- } \\
\text { Moderate- } \\
\text { Heavy) }\end{array}$ & $\begin{array}{l}\text { Physical activity } \\
\text { (Not active- } \\
\text { Moderate- } \\
\text { Active) }\end{array}$ \\
\hline 1 & 15 & $67 \%$ & $33.2 \pm 9.0$ & $9.8 \pm 5.3$ & $7.7 \pm 1.0$ & $0.71 \pm 0.22$ & $5.0 \times / \div 1.7$ & 1.80 & $75.4 \pm 8.0$ & $73--7--20 \%$ & $67--27--6 \%$ \\
\hline 2 & 35 & $71 \%$ & $38.9 \pm 10.4$ & $21.1 \pm 9.8$ & $8.3 \pm 1.2$ & $0.64 \pm 0.19$ & $3.9 \times / \div 1.8$ & $1.75 \pm 8$ & $73.9 \pm 10.1$ & 65--0--35\% & 70--27--3\% \\
\hline $3--6$ & 15 & $73 \%$ & $37.5 \pm 6.3$ & $21.4 \pm 6.2$ & $9.1 \pm 0.9$ & $0.67 \pm 0.14$ & $5.1 \times / \div 1.6$ & $1.75 \pm 10$ & $78.5 \pm 11.7$ & $54--6--40 \%$ & $65--31--4 \%$ \\
\hline \multicolumn{2}{|c|}{ ANOVA } & NS & NS & $<0.01$ & $<0.01$ & NS & NS & NS & NS & NS & NS \\
\hline
\end{tabular}

Data are mean \pm SD except for UAE presented as geometric mean $\times / \div$ tolerance factor

Table 2. Ambulatory blood pressure and heart rate

\begin{tabular}{|c|c|c|c|c|c|c|c|c|c|c|c|c|}
\hline \multirow{2}{*}{$\begin{array}{l}\text { Eye } \\
\text { category }\end{array}$} & \multicolumn{4}{|c|}{ Systolic AMBP (mm Hg) } & \multicolumn{4}{|c|}{ Diastolic AMBP (mm Hg) } & \multicolumn{4}{|c|}{ Heart rate (beats/min) } \\
\hline & 24-h & Day & Night & $\begin{array}{l}\text { N/D ratio } \\
(\%)\end{array}$ & 24-h & Day & Night & $\begin{array}{l}\text { N/D ratio } \\
(\%)\end{array}$ & 24-h & Day & Night & $\begin{array}{l}\text { N/D ratio } \\
(\%)\end{array}$ \\
\hline 1 & $120 \pm$ & $125 \pm$ & $8 \pm 6$ & 86.1 & 72 & $77 \pm 6$ & $61 \pm$ & $79.1 \pm$ & 73 & 77 & $63=$ & 80.4 \\
\hline 2 & $4 \pm 10$ & $129 \pm 10$ & $113 \pm 11$ & 87.6 & $75 \pm 6$ & $80 \pm 6$ & & 81 & $76 \pm 7$ & 81 & 66 & $81.3 \pm 8$ \\
\hline $3--6$ & & $128 \pm 11$ & $114 \pm 8$ & $88.9 \pm 5$ & $76 \pm 7$ & $80 \pm 7$ & $68 \pm 8$ & $84.6 \pm 4$ & $80 \pm 10$ & $86 \pm 10$ & $69 \pm 11$ & $80.3 \pm 6$ \\
\hline ANOVA & NS & NS & NS & NS & NS & NS & 0.02 & 0.02 & 0.11 & 0.07 & NS & NS \\
\hline
\end{tabular}

Data are means \pm SD

obtained at 20-min intervals throughout $24 \mathrm{~h}$. Measurements were performed during a day with normal activities at home or at work. Individually reported sleeping times were implemented in the calculation of day and night blood pressure. Records with more than 2 missing hours were excluded (two patients).

Statistical analysis. Before analysis, UAE values were $\log$ transformed to approximate normal distribution. When analysis of variance (ANOVA) indicated significant differences between groups, pairwise comparisons were assessed with significance levels appropriately modified using the method of Bonferroni. For non-continuous variables the chi-square test with Yates correction was used. A two-tailed $p$ value of less than 0.05 was considered significant. Results are expressed as mean \pm SD except for UAE, which is presented as geometric mean $\times 1$

$\div$ tolerance factor.

\section{Results}

Fifteen patients had no detectable retinal changes (grade 1), 35 had grade 2 retinopathy, and 15 had more advanced retinopathy (grades 3--6). The latter were grouped together due to the small numbers in each group [9]. Clinical characteristics of the patients when grouped according to severity of retinopathy are given in Table 1 . The mean $\mathrm{HbA}_{1 \mathrm{c}}$ increased significantly with increasing levels of retinopathy $(p<0.01)$.The patients were similar regarding UAE, smoking habits, level of physical activity, and insulin consumption. Duration of diabetes was significantly longer in patients in category 2 and 3--6 compared to patients with no retinopathy and body mass index was slightly higher in patients in category 3--6 com- pared to patients with no retinopathy. Data on AMBP and heart rate are given in Table 2. AMBP was consistently higher in the groups with retinopathy (group 2 and 3--6) compared to the group with no detectable retinal changes, reaching statistical significance for diastolic night blood pressure $(p<0.02)$. Furthermore, diurnal variation was significantly blunted in the patients with retinopathy as indicated by a higher night/day ratio of diastolic blood pressure $(p<0.01)$. Individual values of diastolic night blood and diastolic night/day ratio for the three groups are depicted in Figure 1 and 2. As apparent from the figures there was a stepwise increase in both diastolic night blood pressure and diastolic night/day ratio with increasing eye involvement and tests for linear trend were statistically significant for both night blood pressure and night/day ratio $(p<0.01$ for both). Heart rate tended to be higher in patients in group 2 and 3--6 compared to patients without retinopathy with $p$ values of less than 0.06 and 0.11 for day-time and $24 \mathrm{~h}$, respectively. There was no association between duration of diabetes and circadian blood pressure variation $(r=0.1, p=0.66)$, and the differences in night blood pressure and circadian variation persisted after entering diabetes duration as a covariate in the ANOVA, thus adjusting for the differences in duration between the groups $(p<0.02$ for the effect of retina grade). As the three groups were significantly different in respect to metabolic control, we analysed whether part of the differenses in blood pressure and circadian blood pressure variation was explained by differences in $\mathrm{HbA}_{1 \mathrm{c}}$. There was no correlation between $\mathrm{HbA}_{1 \mathrm{c}}$ and diastolic night blood pressure or diastolic night/day ratio $(r=0.15$ 


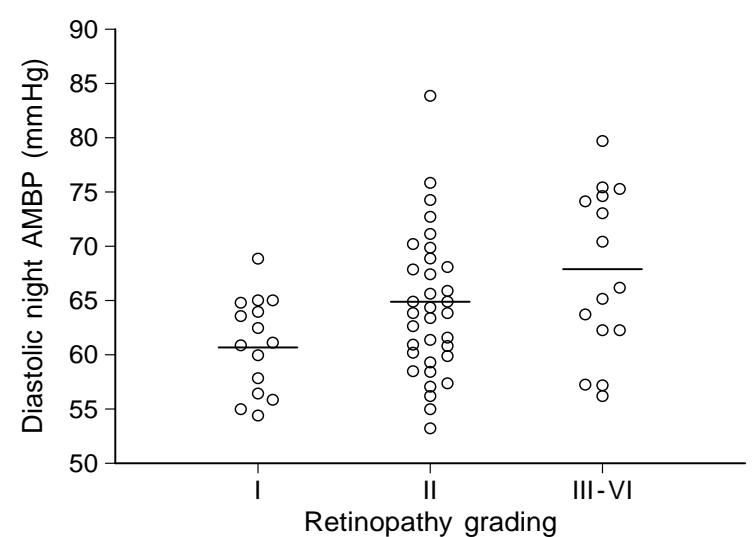

Fig. 1. Diastolic night ambulatory blood pressure according to retinopathy grading (bars indicate mean values)

and $0.10 p>0.40$ for both), and when $\mathrm{HbA}_{1 \mathrm{c}}$ was included as a covariate in the ANOVA, the differences in both diastolic night blood pressure and diastolic night/day ratio persisted $(p<0.05$ and $p<0.03$, respectively). When in addition to $\mathrm{HbA}_{1 \mathrm{c}}$, weight, age, duration, and cigarette consumption were included as covariates, the effect of retinal stage on diastolic night/day ratio barely reached statistical significance $(p=0.07)$.

Comparing patients without diabetic retinopathy (group 1, $n=15$ ) with patients with all grades of retinopathy (group 2 and group 3--6, $n=50$ ) showed higher diastolic night blood pressure (61 \pm 4 compared to $66 \pm 7 \mathrm{mmHg}, p<0.01)$ and higher diastolic night/ day ratio $(79.1 \pm 4$ vs $82.2 \pm 6 \%, p<0.03)$ in the group with diabetic retinopathy. In addition, 24-h systolic and diastolic AMBP also tended to be higher in the group with diabetic retinopathy compared to patients without diabetic retinopathy: $124 \pm 10 / 75 \pm 6$ vs $120 \pm 7 / 72 \pm 5 \mathrm{mmHg}, p=0.07$ and $p=0.09$, respectively.

\section{Discussion}

We present data for a group of patients examined with 24-h AMBP -- a technique not previously implemented in the study of retinopathy in IDDM. In comparison with clinical blood pressure measurement, 24-h AMBP offers several advantages: a multitude of measurements, a lower intrapatient variation [33], a superior correlation with both the degree of targetorgan lesions in essential hypertensive patients [34] and with microalbuminuria in IDDM patients [25]. Moreover, AMBP provides an integrated blood pressure profile over time and includes night measurements, thus allowing for an exploration of circadian blood pressure patterns. In IDDM patients with diabetic nephropathy, cross-sectional studies have shown disturbances in diurnal variation characterized by a blunted diurnal rhythm, and this has been shown

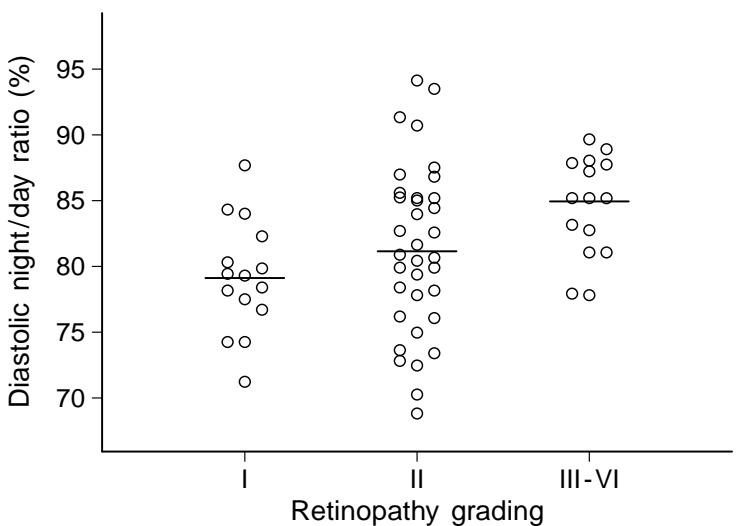

Fig. 2. Diastolic night ratio according to retinopathy grading (bars indicate mean values)

to increase when diabetic nephropathy increases from microalbuminuria to overt nephropathy [35]. The patients included in the present study all had normal clinical blood pressure and normal. In spite of this, we found higher night blood pressure and blunted diurnal variation (increased night/day ratio) with increasing levels of retinopathy. Thus, the two major complications of IDDM, nephropathy and retinopathy, seem to share this pathophysiological feature. The groups were similar regarding age, sex, and level of physical activity. Notably, UAE was almost identical in the three groups excluding this potential confounder. Furthermore, we found an association between poorer glycaemic control as indicated by higher $\mathrm{HbA}_{1 \mathrm{c}}$ and retinopathy. Our data could be seen in context with the haemodynamic hypothesis proposed by Patel and Kohner [36, 37]: blood pressure elevation increases perfusion pressure, and in the presence of hyperglycaemia, the autoregulatory adaption of vessels is inhibited. This induces hyperperfusion, which by increasing shear stress leads to capillary damage and closure, causing retinal ischaemia with subsequent additional hyperperfusion. The higher heart rate in patients with retinopathy would also -ceteris paribus -- tend to increase perfusion. Although both day, 24-h, and night blood pressure numerically were higher with higher grades of retinopathy, only night blood pressure reached statistical significance. It is noteworthy that an association between impaired reduction of night blood pressure has been described for other organ lesions (left ventricular cardiac mass [38], cardiovascular episodes in women [39]).

The combination of higher heart rate and blunted diurnal blood pressure variation with disproportionate elevation of night blood pressure could also indicate autonomic dysfunction in patients with retinopathy, and a connection between autonomic dysfunction and poor autoregulation of retinal vessels could be considered [40]. In addition, the risk of early onset proliferative retinopathy has been reported to be closely related to cardiovascular autonomic neuropa- 
thy [41]. It is however important to notice that the retinal vessels, unlike most other vessels in the circulation do not possess any functioning sympathetic innervation [42]. Thus, neuropathy may be a risk indicator of yet unknown aetiologic processes underlying the development of diabetic retinopathy. Sinclair et al. [43] has described increasing impairment in autoregulation of retinal blood flow with increasing grades of diabetic retinopathy. This produces a situation, where even discrete elevations in systemic blood pressure are readily transmitted to the retinal microcirculation, resulting in capillary hypertension and hyperperfusion. In a study by Parving et al. [44] the effect of antihypertensive treatment (ACE inhibitors and diuretics) on blood-retinal barrier leakage was assessed. The results suggested that systemic blood pressure elevation contributes to an increased leakage of fluorescein across the blood-retinal barrier, and that this leakage can be reversed by antihypertensive treatment. No controlled studies evaluating the effect of antihypertensive treatment on the initiation and progression of diabetic retinopathy have been performed, although preliminary reports [44, 45] seem to indicate a possible positive effect.

Nlorgaard et al. [46] attempted to separate the possible effects of hypertension and of increased urinary albumin loss on diabetic retinopathy by comparing hypertensive, normoalbuminuric IDDM patients with normotensive, normoalbuminuric patients and found no statistically significant difference in prevalence of diabetic retinopathy, thus claiming that hypertension per se is not associated with increased retinal changes. However, since the conclusion is based on a negative finding, the risk of a type 2 error must be taken into consideration; indeed the prevalence of advanced retinopathy was $17 \%$ among the hypertensive patients $(n=51)$ compared to $9 \%$ among the normotensive patients $(n=55)$. Furthermore, the methods used for eye examination (ophthalmoscopy by physicians) and blood pressure measurements (single standard sphygmomanometer measurements to the nearest $5 \mathrm{mmHg}$ ) may also add to the risk of missing a difference.

In a recent large population-based study employing retinal photographs, diastolic blood pressure remained a significant risk factor for moderate-severe retinopathy in IDDM after adjusting for age, duration, $\mathrm{HbA}_{1 \mathrm{c}}$, and UAE [47]. As indicated in our analysis taking cigarette smoking and $\mathrm{HbA}_{1 \mathrm{c}}$ into account it is possible that part of the blood pressure elevation is explained by these two factors. However, in the light of the well-known difficulties in obtaining better glycaemic control and smoking cessation we think that the finding of higher blood pressure is potentially important from a clinical point of view.

In non-diabetic patients with mild essential hypertension, Dimmitt et al. [48] found no association between AMBP and retinopathy assessed by fundal photographs. However, only daytime measurements were performed and potential differences in night blood pressure could thus not be accounted for. In a population-based study [49] Stephenson et al. recently described a retinopathy-dependent difference in the relationship between blood pressure and UAE: in IDDM patients with retinopathy UAE increased steeply with blood pressure as opposed to those without retinopathy who exhibited virtually no rise in UAE with increasing blood pressure.

In conclusion, our data suggest that in IDDM patients, the relationship between retinopathy and blood pressure may not be confined to patients with elevated UAE but could be present independent of renal disease. Prospective studies are needed in order to further evaluate the association between blood pressure elevation and diabetic retinopathy and controlled studies designed to evaluate whether antihypertensive therapy may impede or prevent development and progression of diabetic retinopathy would be of interest.

\section{References}

1. Klein R, Klein BE, Moss SE, Davis MD, DeMets DL (1984) The Wisconsin epidemiologic study of diabetic retinopathy. II. Prevalence and risk of diabetic retinopathy when age at diagnosis is less than 30 years. Arch Ophthalmol 102: 520--526

2. Orchard TJ, Dorman JS, Maser RE, et al. (1990) Prevalence of complications in IDDM by sex and duration. Pittsburgh Epidemiology of Diabetes Complications Study II. Diabetes 39: $1116--1124$

3. Danielsen R, Jonasson F, Helgason T (1982) Prevalence of retinopathy and proteinuria in type 1 diabetics in Iceland. Acta Med. Scand. 212: 277--280

4. Dwyer MS, Melton LJ, Ballard DJ, Palumbo PJ, Trautmann JC, Chu CP (1985) Incidence of diabetic retinopathy and blindness: a population-based study in Rochester, Minnesota. Diabetes Care 8: 316--322

5. Moss SE, Klein R, Klein BE (1988) The incidence of vision loss in a diabetic population. Ophthalmology 95: 1340--1348

6. Vision problems in the US (1980) Data analysis: definitions, data sources, detailed data tables, analysis, interpretation. National Society to Prevent Blindness, New York, NY

7. Klein R, Klein BE, Moss SE, Cruickshanks KJ (1994) Relationship of hyperglycemia to the long-term incidence and progression of diabetic retinopathy. Arch Intern Med 154: 2169-2178

8. The Diabetes Control and Complication Trial Research Group (1993) The effect of intensive treatment of diabetes on the development and progression of long-term complications in insulin-dependent diabetes mellitus. N Engl J Med 329: 977--986

9. Chase HP, Garg SK, Jackson WE et al. (1990) Blood pressure and retinopathy in type I diabetes. Ophthalmology 97: 155-159

10. Knuimann MW, Welborn TA, McCann VJ, Stanton KG, Constable IJ (1986) Prevalence of diabetic complications in relation to risk factors. Diabetes 35: 1332--1339

11. Mathiesen ER, Hommel E, Giese J, Parving H-H (1991) Efficacy of captopril in postponing nephropathy in normotensive insulin dependent diabetic patients with microalbuminuria. BMJ 303: 81--87 
12. Marre M, Chatellier G, Leblanc H, Guyene TT, Menard J, Passa P (1988) Prevention of diabetic nephropathy with enalapril in normotensive diabetics with microalbuminuria. BMJ 297: 1092--1095

13. Viberti G, Mogensen CE, Groop LC, Pauls JF (1994) Effect of captopril on progression to clinical proteinuria in patients with insulin-dependent diabetes mellitus and microalbuminuria. European Microalbuminuria Captopril Study Group. JAMA 271: 275--279

14. Mogensen CE (1982) Long-term antihypertensive treatment inhibiting progression of diabetic nephropathy. Br Med J Clin Res Ed 285: 685--688

15. Parving HH, Andersen AR, Smidt UM, Svendsen PA (1983) Early aggressive antihypertensive treatment reduces rate of decline in kidney function in diabetic nephropathy. Lancet 1 : 1175--1179

16. Parving H-H, Smidt UM, Hommel E et al. (1993) Effective antihypertensive treatment postpones renal insufficiency in diabetic nephropathy. Am J Kidney Dis 22: 188--195

17. Anderson S, Brenne BM (1988) Influence of antihypertensive therapy on development and progression of diabetic glomerulopathy. Diabetes Care 11: 846--849

18. Agardh E, Agardh CD, Torffvit O (1994) A 5-year follow-up study on the incidence of retinopathy in type 1 diabetes mellitus in relation to medical risk indicators. J Intern Med 235: 353--358

19. Moss SE, Klein R, Klein BE (1994) Ocular factors in the incidence and progression of diabetic retinopathy. Ophthalmology 101: 77--83

20. Sawicki PT, Muhlhauser I, Didjurgeit U, Baumgartner A, Bender R, Berger M (1995) Intensified antihypertensive therapy is associated with improved survival in type 1 diabetic patients with nephropathy. J Hypertens 13: 933--938

21. Mathiesen ER, Oxenboll B, Johansen K, Svendsen PA, Deckert T (1984) Incipient nephropathy in type 1 (insulin-dependent) diabetes. Diabetologia 26: 406--410

22. Wiseman M, Viberti G, Mackintosh D, Jarrett RJ, Keen $\mathrm{H}$ (1984) Glycaemia, arterial pressure and micro-albuminuria in type 1 (insulin-dependent) diabetes mellitus. Diabetologia 26: 401--405

23. Feldt Rasmussen B, Borch-Johnsen BK, Mathiesen ER (1985) Hypertension in diabetes as related to nephropathy. Early blood pressure changes. Hypertension 7:II18--II20

24. Joner G, Brinchmann Hansen O, Torres CG, Hanssen KF (1992) A nationwide cross-sectional study of retinopathy and microalbuminuria in young Norwegian type 1 (insulin-dependent) diabetic patients. Diabetologia 35: 1049--1054

25. Hansen KW, Christensen CK, Andersen PH, Pedersen MM, Christiansen JS, Mogensen CE (1992) Ambulatory blood pressure in microalbuminuric type 1 diabetic patients. Kidney Int 41: 847--854

26. Poulsen PL, Hansen KW, Mogensen CE (1994) Ambulatory blood pressure in the transition from normo- to microalbuminuria. A longitudinal study in IDDM patients. Diabetes 43: $1248--1253$

27. Berrut G, Hallab M, Bouhanick B, Chameau AM, Marre M, Fressinaud P (1994) Value of ambulatory blood pressure monitoring in type 1 (insulin-dependent) diabetic patients with incipient diabetic nephropathy. Am J Hypertens 7: 222--227

28. Christensen CK, Mogensen CE (1985) The course of incipient diabetic nephropathy: studies of albumin excretion and blood pressure. Diabet Med 2: 97--102

29. Feldt Rasmussen B, Mathiesen ER, Deckert T (1986) Effect of two years of strict metabolic control on progression of incipient nephropathy in insulin-dependent diabetes. Lancet II: 1300--1304

30. Early treatment for diabetic retinopathy study group (1991) Grading diabetic retinopathy from stereoscopic color fundus photographs -- an extension of the modified Airlie House clas- sification. ETDRS report number 10. Ophthalmology 98[Suppl]:786--806

31. Early treatment for diabetic retinopathy study group (1991) Fundus photographic risk factors for progression of diabetic retinopathy. ETDRS Report number 12. Ophthalmology 98: 823--833

32. O'Brien E, Mee F, Atkins N, O'Malley K (1991) Accuracy of the SpaceLabs 90207 determined by the British Hypertension Society protocol. J Hypertens 9: 573--574

33. Coats AJS, Conway J, Somers VK (1989) Ambulatory pressure monitoring in the assessment of antihypertensive therapy. Cardiovasc Drugs Research 3: 303--311

34. Solokow M, Werdegar D, Kain HK (1966) Relationship between level of blood pressure measured casually and by portable recorders and the severity of complications in essential hypertension. Circulation 34: 279--298

35. Hansen KW, Mau Pedersen M, Marshall SM, Christiansen JS, Mogensen CE (1992) Circadian variation of blood pressure in patients with diabetic nephropathy. Diabetologia 35: 1074-1079

36. Patel V, Rassam S, Newsom R, Wiek J, Kohne E (1992) Retinal blood flow in diabetic retinopathy. BMJ 305: 678--683

37. Kohner EM, Patel V, Rassam SM (1995) Role of blood flow and impaired autoregulation in the pathogenesis of diabetic retinopathy. Diabetes 44: 603--607

38. Verdecchia P, Schillaci G, Guerrieri M, Gatteschi C, Benemio G, Boldrini F, Porcellati C (1990) Circadian blood pressure changes and left ventricular hypertrophy in essential hypertension. Circulation 81: 528--536

39. Verdecchia P, Porcellati C, Schillaci G et al. (1994) Ambulatory blood pressure. An independent predictor of prognosis in essential hypertension. Hypertension 24: 793--801

40. Lanigan LP, Clark CV, Allawi J, Hill DW, Keen H (1990) Impaired autoregulation of the retinal vasculature and microalbuminuria in diabetes mellitus. Eye 4: 174--180

41. Krolewski AS, Barzilay J, Warram JH, Martin BC, Pfeifer M, Rand LI (1992) Risk of early-onset proliferative retinopathy in IDDM is closely related to cardiovascular autonomic neuropathy. Diabetes 41: 430--437

42. Laties AM (1967) Central retinal artery innervation. Absence of adrenergic innervation to the intraocular branches. Arch Opthalmol 77: 405--409

43. Sinclair SH, Graunvald JE, Riva C, Braunstein SL, Nichols CW, Schwartz S (1982) Retinal vascular autoregulation in diabetes mellitus. Ophthalmology 89: 748--750

44. Parving H-H, Larsen M, Hommel E, Lund Andersen H (1989) Effect of antihypertensive treatment on blood-retinal barrier permeability to fluorescein in hypertensive type 1 (insulin-dependent) diabetic patients with background retinopathy. Diabetologia 32: 440--444

45. The EUCLID Study Group (1997) The effect of lisinopril on retinopathy in people wtih insulin dependent diabetes mellitus (IDDM). (Abstract). Diabetologia 40 [Suppl. 1] A 500

46. Norgaard K, Feldt Rasmussen B, Deckert T (1991) Is hypertension a major independent risk factor for retinopathy in type 1 diabetes? Diabet Med 8: 334--337

47. Sjolie AK, Stephenson JM, Aldington S et al. (1997) Retinopathy and vision loss in insulin-dependent diabetes in Europe. The EURODIAB IDDM complications study. Ophthalmology $104: 252--260$

48. Dimmitt SB, West JN, Eames SM, Gibson JM, Gosling P, Little WA (1989) Usefulness of ophthalmoscopy in mild to moderate hypertension. Lancet 1: 1103--1106

49. Stephenson JM, Fuller JH, Viberti GC, Sjolie AK, Navalesi R (1995) Blood pressure, retinopathy and urinary albumin excretion in IDDM: the EURODIAB IDDM Complications Study. Diabetologia 38: 599--603 